\title{
Walking activities and wear of prostheses
}

\author{
B B SEEDHOM ${ }^{1}$ AND N C WALLBRIDGE ${ }^{2}$ \\ From the ${ }^{1}$ Rheumatism Research Unit, and the ${ }^{2}$ Department of Mechanical Engineering, Leeds University
}

SUMmARY A study of the walking activities of 243 individuals was carried out. The individuals $\vec{\circ}$ came from four different occupations and had an age range of 17-83 years. The survey carried out in this investigation showed surprisingly little correlation between variables such as age, height, and weight of individuals and their speed of walking, length of stride, or distance walked. $\frac{\bar{\alpha}}{0}$ Correlation matrices were obtained for the whole sample and then for each sex, showing similar trends. The most significant correlation was between the height of an individual and the length of the stride, and there was a lesser correlation between age and the number of steps walked by anio individual per day. Further statistical analyses showed that males in manual occupations walked $\mathscr{\omega}_{0}$ most and those in sedentary occupations walked least. On the other hand, in the female groups? housewives seemed to walk least and those in technical occupations walked most. The averagenumber of steps walked per day by a male individual for the whole sample was 9537 ; that forष females was 9839 . The corresponding distances walked per day were 6.7 and $6.5 \mathrm{~km}$. The@ differences were not statistically significant. Predictions of wear of prosthetic components made of ultrahigh molecular weight polyethylene were made on the basis of the above data and other ${ }^{\circ}$ variables affecting wear, such as the weight of the subject and the area available for cont $\overrightarrow{0}$ during walking. Charts have been constructed of the penetration of the metallic component intor the plastic one for both hip and knee prostheses, thus enabling predictions of the wear of the plastic components of these two most widely used prostheses. Owing to the wide ranging valuess of the variables used in making the predictions of wear, these latter should be regarded only aso 'safe' first estimates.

Key words: daily walking distance, wear, prosthetic hips and knees.

Walking is the most predominant of the locomotive activities of humans and perhaps the most predominant of Western man's activities. Much fewer cycles of sitting, kneeling, or squatting occur in Western Societies than in the Eastern. Walking is therefore the most important activity from the point of view of wear of lower limb prosthetic joints. During this process removal of particles from the articulating surfaces of the prosthesis occurs, and if the amount of debris removed is large tissue reaction may result. Or else, as a consequence of excessive wear the mechanical strength of the prosthetic component in question may be significantly reduced such that it will fail under the large loads arising in the joint during walking.

It is interesting that relatively few data are

Accepted for publication 1 May 1985.

Correspondence to Dr B B Seedhom, Rheumatology and Rehabilitation, School of Medicine, 36 Clarendon Road, Leeds LS2 9PJ. published on the subject of walking activities. This is perhaps due to the difficulty of obtaining measure ments from a large number of individuals and partlyo due to popular assumptions concerning this matter 3 It is a popular view that certain correlations exist? for instance that the shorter a person the faster the pace, and the shorter the stride will be; or, the heavier or older an individual, the slower will be the pace of walking etc.

This communication is concerned with a survey of a number of the above mentioned variables in relatively large sample of individuals from different occupations and with the effect of some of these variables on the wear of two of the most implante prostheses, the hip and the knee.

\section{Materials and methods}

Two hundred and forty-three subjects were studied Their age, sex, and occupation were noted. Th\&्ठ 
height and body mass were recorded. Their normal cadence of walking was measured by noting how many paces they took in a minute. Then measurements took place in the street or in corridors in work places, such as hospitals, factories, or schools. The stride length was measured by dividing the distance walked by the corresponding number of paces taken. A pedometer was attached to the subject to record the number of steps they walked a day. The subject was asked to wear this pedometer for 24 hours begining at whatever time the pedometer was given to him. The measurements were made during a normal working day from Monday to Friday.

The data were subjected to statistical analysis to obtain the means and standard deviations and to investigate further: $(a)$ if correlations existed between some of the measured quantities and $(b)$ if there were significant differences between the sexes and groups within the different occupations. The yearly wear rate of hip acetabular cups and knee tibial components commonly made of ultrahigh molecular weight polyethylene (UHMWPE) was predicted on the basis of the relevant data obtained.

Table 1 Classification of participants according to sex and occupation

\begin{tabular}{lcl}
\hline Occupation & Male & Female \\
\hline Sedentary & 53 & 22 \\
Technical & 35 & 27 \\
Manual & 56 & 19 \\
Housewives & - & 31 \\
Total & 144 & 99 \\
\hline
\end{tabular}

Table 2 Age, height, and body mass distribution in all participants

\begin{tabular}{|c|c|c|c|c|c|c|}
\hline & \multicolumn{3}{|l|}{ Male } & \multicolumn{3}{|c|}{ Female } \\
\hline & Mean & $S D$ & Range & Mean & $S D$ & Range \\
\hline Age (years) & 44.9 & $15 \cdot 7$ & $17-78$ & $40 \cdot 7$ & $15 \cdot 8$ & $17-83$ \\
\hline Height $(\mathrm{cm})$ & $174 \cdot 5$ & $7 \cdot 5$ & $157-193$ & $161 \cdot 7$ & $5 \cdot 4$ & $150-178$ \\
\hline Body mass (kg) & 72 & $8 \cdot 8$ & $49 \cdot 3-103 \cdot 5$ & $63 \cdot 2$ & $8 \cdot 5$ & $41-96$ \\
\hline
\end{tabular}

\section{Results and discussion}

Of the participants, 144 were male and 99 were female. The classification of occupations is shown in Table 1.

The means, standard deviations, and ranges of age (years), height (centimetres), and body mass (kilograms) are shown in Table 2. The means, standard deviations, and ranges of cadence (strides/ minute), stride length $(\mathrm{cm})$, number of steps per day, and the corresponding distance walked per day are shown in Table 3. The data in Tables 1-3 are for the male and female sample from all occupations. Similar data have been obtained for each occupation group for both sexes. These are presented in Tables 4-7.

Table 4 Statistical data on persons in sedentary occupations

\begin{tabular}{llll}
\hline & & $\begin{array}{l}\text { Male } \\
(n=53)\end{array}$ & $\begin{array}{l}\text { Female } \\
(n=22)\end{array}$ \\
\hline Cadence (strides/min) & Mean & $62 \cdot 3$ & $63 \cdot 7$ \\
& SD & $10 \cdot 8$ & $12 \cdot 8$ \\
Stride length (cm) & Mean & $71 \cdot 4$ & $62 \cdot 9$ \\
& SD & $5 \cdot 5$ & $4 \cdot 7$ \\
No of steps/day & Mean & 8241 & 7954 \\
& SD & 3911 & 5997 \\
Distance walked/day (km) & Mean & $5 \cdot 93$ & $5 \cdot 10$ \\
& SD & $3 \cdot 09$ & 3.74 \\
\hline
\end{tabular}

Table 5 Statistical data on persons in technical occupations

\begin{tabular}{llll}
\hline & & $\begin{array}{l}\text { Male } \\
(n=35)\end{array}$ & $\begin{array}{l}\text { Female } \\
(n=27)\end{array}$ \\
\hline Cadence (strides/min) & Mean & $65 \cdot 5$ & $58 \cdot 5$ \\
& SD & $10 \cdot 6$ & $6 \cdot 3$ \\
Stride length (cm) & Mean & $71 \cdot 2$ & $70 \cdot 0$ \\
& SD & $9 \cdot 8$ & $8 \cdot 3$ \\
No of steps/day & Mean & 10338 & 15433 \\
& SD & 4587 & 5353 \\
Distance walked/day $(\mathrm{km})$ & Mean & $7 \cdot 36$ & $10 \cdot 58$ \\
& SD & $3 \cdot 3$ & $4 \cdot 4$ \\
\hline
\end{tabular}

Table 3 Distribution of cadence, stride length, number of steps and distance walked/day

\begin{tabular}{|c|c|c|c|c|c|c|}
\hline & \multicolumn{3}{|c|}{ Male $(n=144)$} & \multicolumn{3}{|c|}{ Female $(n=99)$} \\
\hline & Mean & $S D$ & Range & Mean & $S D$ & Range \\
\hline Cadence (strides/min) & $63 \cdot 7$ & 11 & $31-89$ & 63 & $11 \cdot 31$ & $21-91$ \\
\hline Stride length $(\mathrm{cm})$ & $71 \cdot 3$ & $6 \cdot 9$ & $41-97$ & 65 & $7 \cdot 8$ & $46-81$ \\
\hline No of steps/day & 9537 & 4761 & $1600-35500$ & 9839 & 5706 & $1200-32600$ \\
\hline Distance walked/day (km) & $6 \cdot 7$ & $3 \cdot 2$ & $1-19 \cdot 6$ & $6 \cdot 5$ & $4 \cdot 1$ & $0 \cdot 6-19 \cdot 8$ \\
\hline
\end{tabular}


Table 6 Statistical data on persons doing manual work

\begin{tabular}{llll}
\hline & & $\begin{array}{l}\text { Male } \\
(n=56)\end{array}$ & $\begin{array}{l}\text { Female } \\
(n=19)\end{array}$ \\
\hline Cadence (strides/min) & Mean & $64 \cdot 1$ & $66 \cdot 0$ \\
& SD & $11 \cdot 6$ & $13 \cdot 1$ \\
Stride length (cm) & Mean & $71 \cdot 2$ & $63 \cdot 5$ \\
& SD & $5 \cdot 8$ & $7 \cdot 6$ \\
No of steps/day & Mean & 10248 & 9242 \\
& SD & 5270 & 3217 \\
Distance walked/day (km) & Mean & $7 \cdot 11$ & $5 \cdot 79$ \\
& SD & 2.92 & 1.95 \\
\hline
\end{tabular}

Table 7 Statistical data on housewives $(n=31)$

\begin{tabular}{llr}
\hline Cadence (strides/min) & Mean & $64 \cdot 7$ \\
& SD & $11 \cdot 2$ \\
Stride length (cm) & Mean & $63 \cdot 3$ \\
& SD & $7 \cdot 3$ \\
No of steps/day & Mean & 6922 \\
& SD & 2867 \\
Distance walked/day (km) & Mean & $4 \cdot 34$ \\
. & SD & $1 \cdot 84$ \\
\hline
\end{tabular}

The matrix of the correlation coefficient was computed for all the subjects, and the data are presented in Table 8 . The same was done for all male and female subjects within each sex group, and since the data were quite similar it was deemed unnecessary to include two more such large tables in this paper.

From Table 3 it can be seen that there are no significant differences between sexes so far as the speed of walking or distance walked, but there seems to be a slight difference between the males and females so far as the stride length and number of steps are concerned; males have a longer stride and females take more steps per day.

The analysis of variance (ANOVA) was carried out on all the groups within both sexes to establish if there were significant differences between these groups. The analysis showed that for all male groups there were significant differences $(p<0.05)$ in the number of steps and distance walked per day.
Similar results were obtained for all female groups, with a higher significance $(\mathrm{p}<<0 \cdot 01)$. Further tests were then carried out showing that males in manual $\vec{F}$ occupations walked the most and, as would be $\stackrel{\oplus}{+}$ expected, those in sedentary activities walked least. On the other hand, in the female groups housewives $\overline{\bar{c}}$. seemed to walk least and those in technical occupa- $\bar{\nabla}$ tions walked most. Table 8 is interesting in that it $\varrho$ shows the difference between reality and popularis views concerning correlations between age or height $\vec{\circ}$ or weight and the speed of walking. Although there is a correlation between height and the stride length, $\vec{\omega}$ it is not large. A lesser degree of correlation exists also between age and the number of steps made perọ? day. The high correlation between the number of $\stackrel{\vec{\perp}}{-}$ steps made and the distance walked is not surpris- $\vec{\sim}$ ing, since the distance walked is the product of the $e_{\infty}$ number of steps made by the length of stride.

WEAR OF PROSTHETIC COMPONENTS

The wear rate of a plastic prosthetic component similar to some extent to that of a stick of chalk. The wear characteristic of chalk is dependent on how $\stackrel{\mathbb{}}{乛}$ hard it is pressed against the blackboard and how faro it is drawn. The wear rate of a plastic prosthetic component is similarly dependent on a wear coefe $-\vec{\varphi}$ cient, which is characteristic of the componen sur material rubbing against the metallic (or ceramis) component. It is also dependent on the force acting? on the joint and the distance through which the $\frac{\partial}{0}$ components slide on each other. A simple relationship governs the volume of debris removed from $\Phi$ the plastic component such that:

$$
\mathrm{V}=\mathrm{kPL}
$$

where $\mathrm{V}=$ the volume of debris removed, $\mathrm{k}=$ the wear coefficient characteristic of the plastic sliding? on metal (or from whatever material the component is made of), $\mathrm{P}=$ the force acting on the two? components, and $\mathrm{L}=$ the sliding distance.

Methods of obtaining the coefficient of wear experimentally have been described, and different $\mathcal{O}$ values for $\mathrm{k}$ have been obtained by various authors for UHMWPE sliding on stainless steel, cobalt 9 chrome alloy, and ceramic surfaces.

Table 8 Correlation coefficients

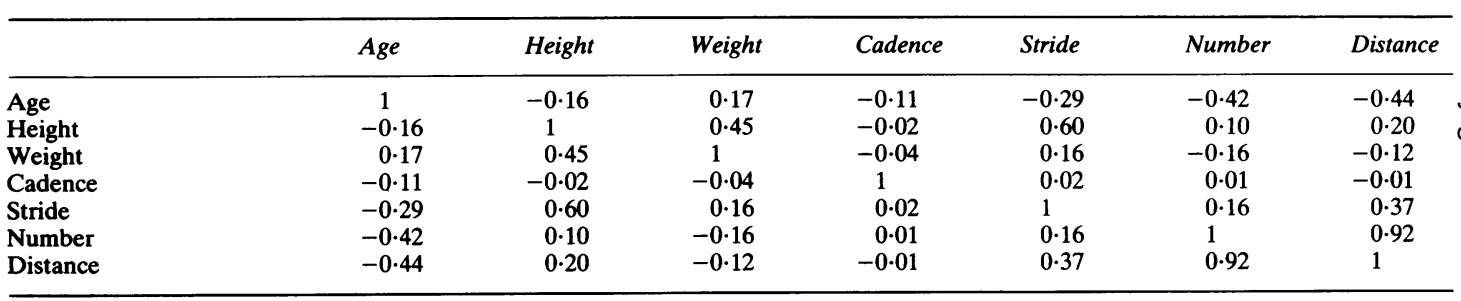


The value of PL, the product of the joint force and the sliding distance, represents the intensity of use of the prosthesis. Since walking is the activity through which a prosthesis is most used, the value of PL is calculated by integration from the load and speed variations with time during one complete walking cycle. The volume of wear debris in a walking cycle can then be calculated. Since a mean value for the number of walking cycles per day taken by various groups is known, the daily volume of wear debris and hence the yearly wear rate of a plastic prosthetic component can be estimated.

It is important to recognise that the calculated yearly wear rate, in terms of the penetration of the metallic component of the prosthesis into the polymeric component, does not take account of the additional contribution to this penetration that might be made by creep of the polymer. Recent estimates of the penetration due to creep and the correlation between these calculated wear and creep penetration rates with in-vivo data for hip joints have been discussed by Downson et al. ${ }^{2 a}$ 2b

It must be noted that the product PL is a function of the weight of the individual, and the heavier the latter, the larger will be the volume of the wear debris. It was interesting to make some theoretical predictions of the wear of different hip and knee prostheses. These predictions, however, were made on the basis of the values of the wear coefficient $k$ and the product PL calculated from published and/or communicated data by Paul ${ }^{1}$ and Morrison. ${ }^{2}$ The following values for the above have been assumed.

1. Wear coefficient values (k) for UHMWPE sliding on stainless steel $6.8 \times 10^{-7} \mathrm{~mm}^{3} / \mathrm{Nm}$. This value is quoted from Dowson et al., ${ }^{3}$ and results from laboratory wear tests under wet conditions with load, speed, and surface finish similar to the use found in prostheses. For UHMWPE, which has been solid phase formed sliding on cobalt chrome alloy the values of $\mathrm{k}$ between 0.86 and $5.9 \times 10^{-7} \mathrm{~mm}^{3} / \mathrm{Nm}$ obtained by Seedhom et al. ${ }^{4}$ will be used here.

2. The load and sliding distance product PL. (a) For a natural hip of diameter $45 \mathrm{~mm}$ and patient of weight $620 \mathrm{~N}(63 \mathrm{~kg}) \mathrm{PL}$ was found to be approximately $50 \mathrm{Nm} .{ }^{4}$ Thus for any artificial hip of radius, $R$ and patient weight, $B$ :

$$
\mathrm{PL}=\frac{50}{620 \times 45} \times 2 \mathrm{RB} \mathrm{Nm}
$$

(b) For a natural knee of an average size and patient of weight $620 \mathrm{~N}(63 \mathrm{~kg}) \mathrm{PL}$ is approximately $60 \mathrm{Nm}^{4}$

For different knee prostheses the value of PL must be calculated (as in the case of the hip prostheses) for the specific geometry and patient weight. Many of knees in current use are of approximately the same radii as those of the natural knee, for example Kinematic, the Freeman Samuelson, the Coutalier, the Leeds, the Geomedic, and others. For these the following relationship is used:

$$
\mathrm{PL}=\frac{60 \mathrm{~B}}{620} \mathrm{Nm}
$$

For other knee prostheses whose femoral and tibial components are much smaller than those of the natural knee, e.g., the Sheehan, a smaller value of PL is assumed:

$$
\mathrm{PL}=\frac{55 \mathrm{~B}}{620} \mathrm{Nm}
$$

The wear rate in the form of penetration of the metallic component into the plastic one in $\mathrm{mm} /$ year is calculated. It is assumed that the wear is uniform on the whole of the contact surface of the plastic component. This is not strictly the case, but it is adopted here as a basis for comparison. Clearly, the approximations used in these calculations limit the accuracy of the results, but some indication of the relative magnitudes of prosthesis wear is obtained.

Fig. 1 represents the wear of hip prostheses

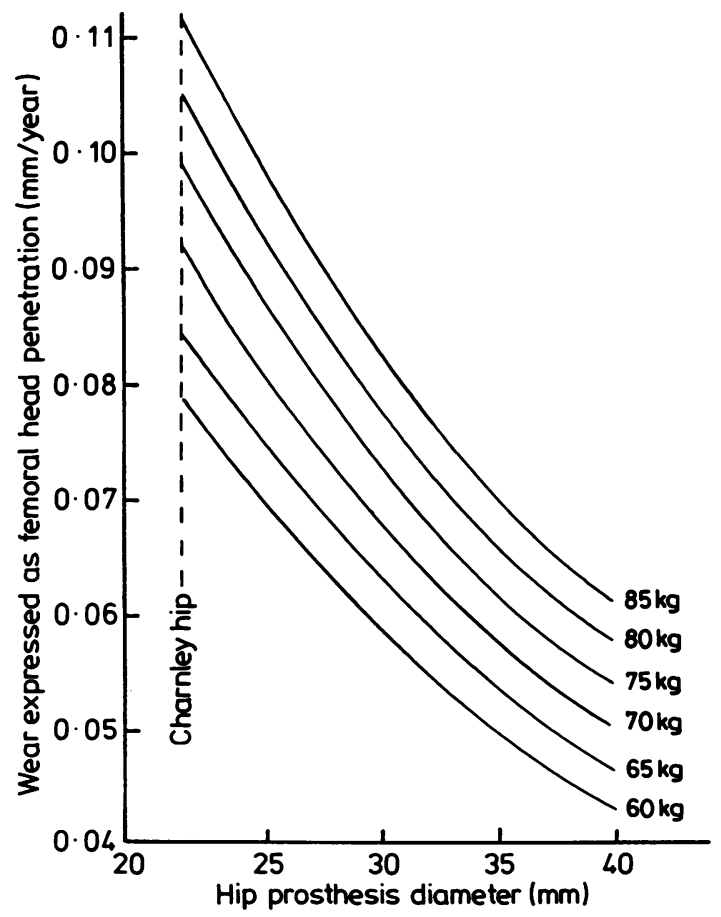

Fig. 1 Wear of hip prosthesis of different diameters, and for individuals of different weights, based on 10000 steps walked by the individual each day. 
expressed as predicted penetration of the femoral component into the acetabular cup in $\mathrm{mm} /$ year. This is calculated for different diameters of the prosthetic femoral heads and also for individuals of body mass between 60 and $85 \mathrm{~kg}$. Fig. 2 represents a different exercise whereby the wear of one hip prosthesis is calculated for individuals within the same weight range and for a range of walking activity between 2000 and 10000 steps per day. As these charts show the predicted penetration of the femoral head in the acetabular cup decreases as the diameter of the femoral head increases. Also, for the same prosthesis, the lighter the patient and the fewer the steps made per day, the lower the year's wear.

The same applies in the case of knee prostheses. The larger the articular area of the prosthesis available for transmitting the loads, the lower the yearly wear, as shown in Fig. 3. The extremes of wear occur in the Sheehan knee, which is typical of a prosthesis with a small contact area, and a prosthesis of the surface replacement type of which the Leeds knee, the Kinematic, the Freeman Samuelson, the Oxford, and others are typical, and all of which have a much larger contact area. These wear rates are plotted for individuals of different weights and for the same range of walking activities, i.e., 200010000 steps per day (Fig. 4).

The above predictions are based on assumptions which the authors wish to discuss.

The pedometers used could conceivably give false/positive registrations corresponding to nonroutine (mainly 'jerky') movements of the leg.

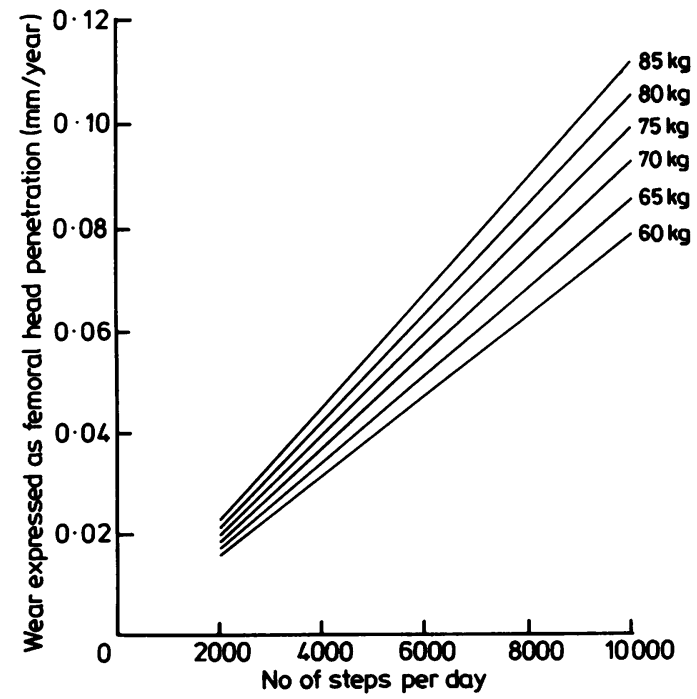

Fig. 2 Wear of Charnley hip prosthesis, for individuals with different body masses and varying walking activities.
These are likely to have only a small effect on the pedometer reading, which can be reasonably assumed to be representative of the daily number of steps taken by the subject.

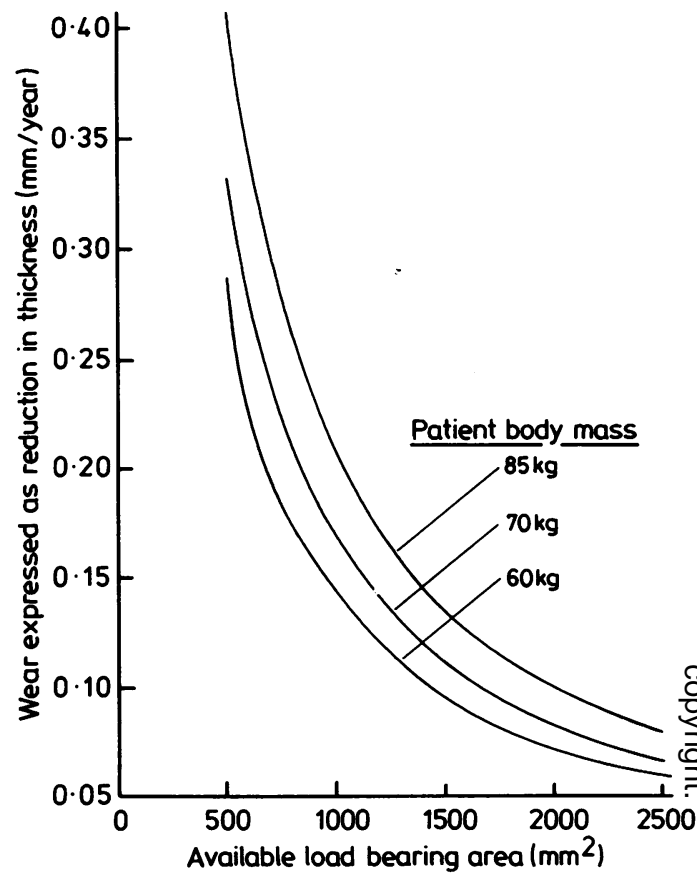

Fig. 3 Wear of knee prosthesis with different areas of the tibial component for individuals with different body masses.

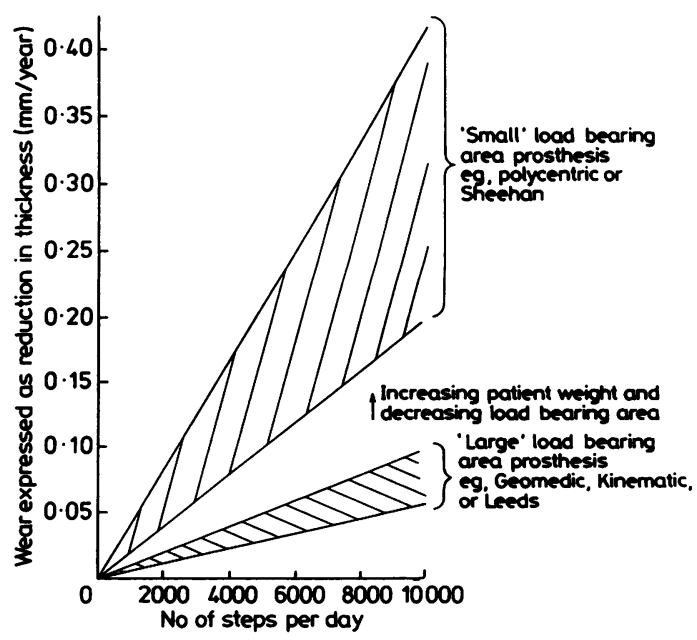

Fig. 4 Wear of knee prosthesis, with varying load bearing areas and patient weights, against patient activity. 
Perhaps the more controversial of the assumptions are those regarding the magnitude of joint forces and number of steps taken per day. Both quantities are expected to be smaller in patients with prosthetic joints than in normals. The predictions of wear have been made on the basis of the larger forces, which may be justified in younger patients whose muscles would still be somewhat vigorous and who may be surprisingly active!

The predictions of wear of the polyethylene prosthetic component must therefore be regarded as 'safe' first estimates, taking into account the wide ranging values of the variables used in the calculations.

The authors wish to thank the different individuals within the different groups who have participated in this survey and those who have helped in collecting the data. In particular, Mr F Pickering, Biology Master at Leeds Grammar School and many of his pupils.

Thanks are also due to Mr Patrick Constable, Department of Statistics and Professor Max Hamilton, Emeritus Professor of Psychiatry, Leeds University for their advice and help on the statistical analyses done in this work.

\section{References}

1 Paul J P. Forces transmitted by joints in the human body. Proceedings of the Institution of Mechanical Engineers. Part 3J. Lubrication and wear in living and artificial human joints. 1967: 181 .

2 Morrison J B. Bioengineering analysis of force actions transmitted to the knee joint. Biomed Eng 1968; 3: 164-70.

2a Dowson D, Wallbridge N C. Laboratory wear tests and clinical observations of the penetration of the femoral heads into acetabular cups in total replacement hip joints. 1. Charnley prosthesis with polytetra flowa ethylene acetabular cups. Wear (in press).

2b Atkinson J R, Dowson D, Isac G $\mathbf{H}$, Wroblewski B $\mathbf{M}$. Laboratory wear tests and clinical observations of the penetration of the femoral heads into acetabular cups in total replacement hip joints. 2. A microscopical study of the surfaces of Charnley polyethylene acetabular sockets in vivo; 3 . The measurement of internal volume changes in explanted Charnley sockets after 2-14 years in vivo and the determination of wear factors. Wear (in press).

3 Dowson D, El-Hady Diab M M, Gillis B J, Atkinson J R. The influence of counterface topography upon the wear of ultra high molecular weight polyethylene under wet or dry conditions. In: Lieng-Huang Lee, ed. Polymer wear and its control. St Louis, USA: American Chemical Society, 1984.

4 Seedhom B B, Dowson D, Wright V. Wear of solid phase formed high density polyethylene in relation to the life of artificial hips and knees. Wear 1973; 24: 35-51. 